\title{
Adenocarcinoma insitu presenting as extensive crazy paving pattern: HRCT findings and review of literature
}

\author{
Dev R. ${ }^{1}$, Sharma P. ${ }^{2}$, Khan Y. ${ }^{3}$ \\ ${ }^{1}$ Dr. Rahul Dev, Assistant Professor, Department of Radiodiagnosis and Imaging, All India Institute of Medical Sciences, \\ Rishikesh, Uttarakhand, ${ }^{2}$ Dr. Pankaj Sharma, Associate Professor, Department of Radiodiagnosis and Imaging, All India \\ Institute of Medical Sciences, Rishikesh, Uttarakhand, ${ }^{3}$ Dr. Yasrab Khan, Senior Resident, Department of \\ Radiodiagnosis, Delhi State Cancer Institute, Dilshad Garden, New Delhi, India.
}

Corresponding Author: Dr. Rahul Dev, Assistant Professor, Department of Radiodiagnosis and Imaging, All India Institute of Medical Sciences, Rishikesh, Uttarakhand. E-mail: rdev8283@gmail.com.

\begin{abstract}
The crazy-paving pattern seen on HRCT is comprised of diffuse areas of ground glass attenuation. This imaging finding has numerous differentials including idiopathic, infectious, neoplastic and other miscellaneous entities. A 52-year-old non-smoker presented with mild to moderate dyspnoea with occasional cough and restrictive pattern on PFT. Chest radiograph showed bilateral diffuse air space opacities with crazy paving pattern on High resolution CT of the lung. This pattern has numerous differentials with pattern of lung involvement, the patient's history and clinical findings being helpful in narrowing the diagnosis.
\end{abstract}

Keywords: Adenocarcinoma in situ, Bulging fissure sign, Crazy paving, CT angiogram sign, Ground glass attenuation, Interlobular septal thickening,

\section{Introduction}

The "crazy-paving" pattern is a finding seen in HRCT imaging of the lung. It consists of scattered or diffuse areas showing a ground glass pattern of attenuation with superimposed interlobular septal thickening. This pattern has ample differentials including idiopathic, infectious, neoplastic, inhalational, and miscellaneous conditions. It is difficult to distinguish these entities radiologically however the patient's clinical history, including presenting complaints can be helpful in reaching towards appropriate diagnosis [1].We report a case of 52 years old non-smoker presenting with complaints of chronic cough. Chest radiograph showed diffuse air space opacification. Subsequently HRCT showeda crazy paving pattern which on histopathology turned out to be adenocarcinoma in situ.

\section{Case Report}

A 52-year man a non-smoker presented with a history of mild to moderate dyspnoea at rest which was present on exertion initially. There is associated cough with occasional expectoration. There was no history of haemoptysis. On physical examination the patient was lean and of average stature. No evidence of cyanosis or digital clubbing was seen. On auscultation there was evidence of bilateral mid and lower zone crackles with left sided rhonchi. Biochemical investigations reveal normal complete blood counts, including platelets and normal urine analysis.

The patient underwent Chest Radiograph followed by HRCT chest for recognition and evaluation of lung pathology. Chest radiograph (Figure 1) revealed diffuse air space opacities involving bilateral lung fields. HRCT lung window on axial (Figure 2), coronal and sagittal planes (Figures 3) revealed evidence of patchy geographical areas of ground glass attenuation with interlobular septal thickening suggestive of crazy paving pattern involving both lungs. The patient underwent trans-bronchial lung biopsy with a histopathological diagnosis of Mucinous Adenocarcinoma in situ. Subsequently the patient underwent combined chemotherapy with the subjective response in terms of symptoms,

\footnotetext{
Manuscript received: $5^{\text {th }}$ October 2018

Reviewed: $16^{\text {th }}$ October 2018

Author Corrected: $25^{\text {th }}$ October 2018

Accepted for Publication: $30^{\text {th }}$ October 2018
} 


\section{Case Report}

however the radiological findings remained more or less the same. Eventually the patient scummed to the lung pathology and died nearly seven months after diagnosis. Our case is unique as the disease is seen in a male patient who had no history of smoking, presenting as extensive multilobar disease having a crazy paving pattern with a clear depiction of CT angiogram, air bronchogram, bulging fissure signs and presence of pseudo cavitation.

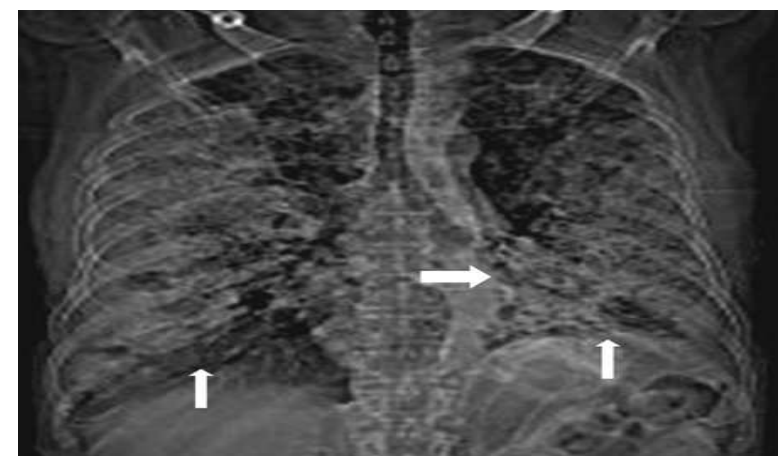

Fig-1: Chest $X$ ray demonstrates diffuse air space opacities involving bilateral lung fields with the fuzzy outline of the left cardiac border (thick white arrow) and bilateral hemidiaphragm outline (thin white arrows).

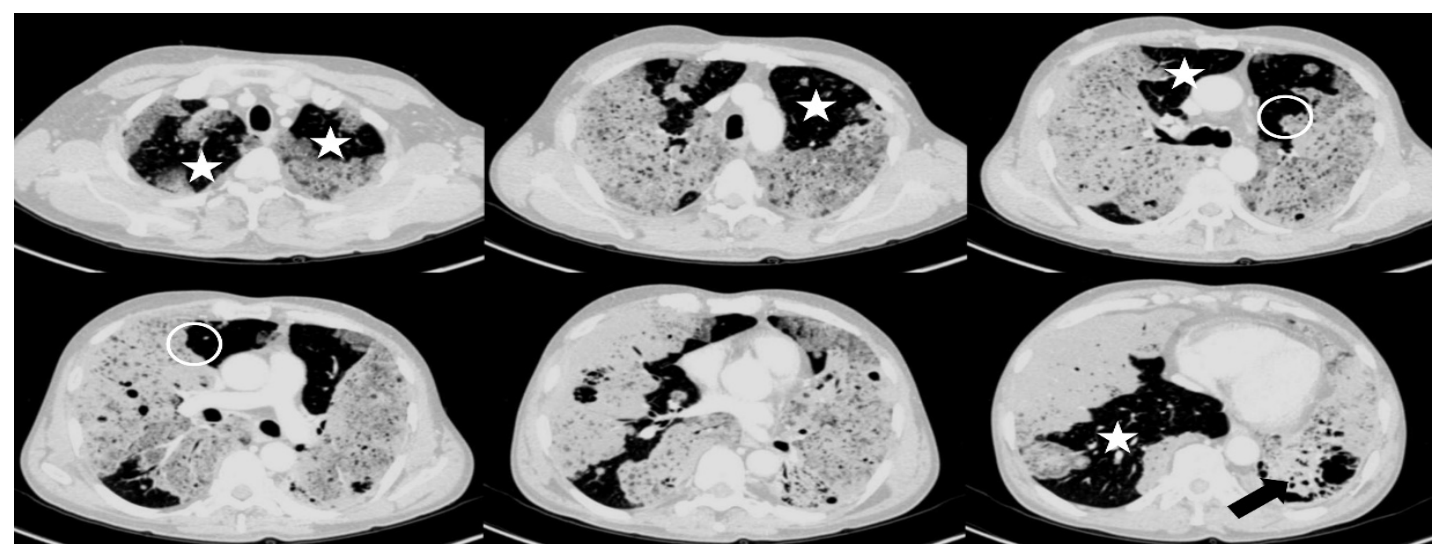

Fig-2: HRCT chest axial sections reveal geographic areas of ground-glass opacity and septal thickening in an asymmetric distribution with relative sparing of apices, anterior segments of upper lobes and right lower lobe (white stars). There is also evidence of collapse of left lower lobe showing atelectobronchiectatic changes (thick black arrow) with mediastinal shift towards left side. Note the sharp demarcation between normal and abnormal lung parenchyma (white circle).

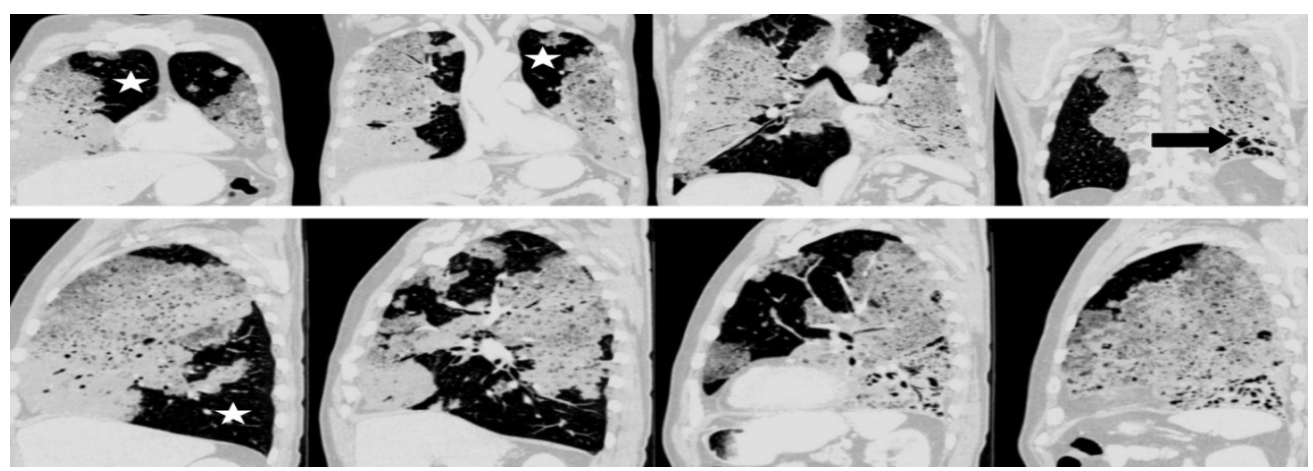

Fig-3: Coronal and Sagittal reformatted images reveal a clearer depiction of spared lung segments (white stars) and left lower lobe collapse (thick black arrow).

\section{Discussion}

The crazy-paving pattern seen on HRCT of the lungs is a nonspecific finding, an appearance of paths made with pieces of stone or concrete. It was first described in patients with pulmonary alveolar proteinosis (PAP) as mentioned by Lee CHin 2007 [2]. This pattern is characterized by alveolar, interstitial or combined 


\section{Case Report}

pathology. This appearance has ample differentials including Idiopathic conditions like Pulmonary Alveolar Proteinosis, nonspecific Interstitial Pneumonia, sarcoidosis, organizing pneumonia, infectious etiologies, including pneumocystis and nonspecific interstitial pneumonia, neoplastic like Adenocarcinoma in situ and inhalational etiologies like lipoid pneumonia [3]. Other miscellaneous conditions include Adult respiratory distress syndrome and pulmonary haemorrhage syndromes. Johkoh et alin 1999 postulated 100\% prevalence of crazy paving pattern in pulmonary alveolar proteinosis with Interstitial pneumonias and ARDS has next higher prevalence rates on the basis of bronchoalveolar lavage or transbronchial lung biopsy with one patient showing the pattern in tubercular etiology [4].

Adenocarcinoma in situ is a subtype of adenocarcinoma with peculiarity of local spread using the lung structure as stroma without underlying architecture distortion. Some of the salient features include peripheral location, scarring, mucin production, high occurrence in nonsmokers and females and multicentricity as enumerated by Trigaux et al in 2006 [5]. Furthermore, it is also being noted that Adenocarcinoma in situ is the cause in the vast majority of cases of adenocarcinoma encountered in recent times. The spectrum of the radiographic findings is broadly divided into solitary lesion, localised consolidation and multicentric or diffuse forms with the consolidative form being most common and extensive multinodular form being the most infrequent pattern [6].

True cavitation is uncommonly seen in Adenocarcinoma in situ due to lack of necrosis and preserved lung framework with normal perfusion though there is presence of pseudo cavitations symbolizing small bronchi. The angiogram sign, although nonspecific been described as a CT sign of Adenocarcinoma in situ defines clear visibility of vessels on post contrast scans.

However, still it may be used as a corroborative finding for suggesting the diagnosis of Adenocarcinoma in situ representing enhancing pulmonary vessels against a background of low attenuation lung parenchyma. Other imaging features include Bulging fissure sign and crazy paving pattern as described by Patsios et al [6].Bulging of the interlobar fissure is caused by mucin production in the tumour resulting in expansion of the lobe whereas crazy paving pattern reflects low-density intra-alveolar material and the superimposed reticular attenuation is caused by interstitial infiltration by inflammatory or tumour cells. The same pattern was seen in our patient.
Pathologically Adenocarcinoma in situ has mucinous and nonmucinous variants with multicentricity and worse prognosis associated with mucinous type as cited in 1997 by Lee et al [7]. Mucinous type is histologically composed of tall columnar cells. Furthermore, Yousem et al in 2007 mentioned there is neither stromal, vascular or pleural invasion nor any nodal disease or extra pulmonary metastasis [8]. As far as prognosis is considered the 5-year survival is highest for nodular form and least for multifocal and consolidative patterns.

Targeted combined chemotherapy has a role in the treatment of Adenocarcinoma in situ with female gender and non-smokers having favourable response. Lung transplantation is a valuable option for patients with respiratory failure secondary to advanced form of multifocal adenocarcinoma in situ as Perrot et alin 2004 mentioned that time frame for disease recurrence and death was significantly longer in patients with Adenocarcinoma in situ than of other variants [9].

\section{Conclusion}

The crazy paving appearance on HRCT is a peculiar, but nonspecific finding to be read in correct clinical context. We report a case of adenocarcinoma in situ seen as crazy paving pattern in a non-smoker male patient.

\section{Funding: Nil, Conflict of interest: None Permission of IRB: Yes}

\section{References}

1. Gardiner N, Jogai S, Wallis A. The revised lung adenocarcinoma classification-an imaging guide. $\mathrm{J}$ Thorac Dis. 2014 Oct; 6 (Suppl 5): S537-46. doi: 10. 3978 /j. issn. 2072-1439.2014.04.05.

2. Lee CH. The crazy-paving sign. Radiology. 2007 Jun; 243(3):905-6. doi:10.1148/radiol.2433041835.

3. Maimon N, Heimer D. The crazy-paving pattern on computed tomography. CMAJ. 2010 Oct 5; 182 (14): 1545. doi: 10.1503/cmaj.091422. Epub 2010 Jun 14.

4. Johkoh T, Itoh H, Müller NL, et al. Crazy-paving appearance at thin-section CT: spectrum of disease and pathologic findings. Radiology. 1999 Apr; 211 (1): 155-60. doi:10. 1148 /radiology. 211. 1. r99 ap 10155.

5. Trigaux JP, Gevenois PA, Goncette L, et al. Bronchioloalveolar carcinoma: computed tomography findings. Eur Respir J. 1996 Jan;9(1):11-6. 


\section{Case Report}

6. Patsios D, Roberts HC, Paul NS, et al. Pictorial review of the many faces of bronchioloalveolar cell carcinoma. Br J Radiol. 2007 Dec; 80 (960):1015-23. Epub 2007 Oct 16.doi:10.1259/bjr/52225107.

7. Lee KS, Kim Y, Han J, et al. Bronchioloalveolar carcinoma: clinical, histopathologic, and radiologic findings. Radiographics. 1997 Nov-Dec; 17 (6):134557. doi:10.1148/radiographics.17.6.9397450.
8. Yousem SA, Beasley MB. Bronchioloalveolar carcinoma: a review of current concepts and evolving issues.ArchPatholLabMed.2007Jul;131(7):1027-32. doi: 10.1043/15432165(2007)131[1027:BCAROC]2.0.CO;2

9. de Perrot M, Chernenko S, Waddell TK, et al. Role of lung transplantation in the treatment of bronchogenic carcinomas for patients with end-stage pulmonary disease. J Clin Oncol. 2004 Nov 1; 22 (21):4351-6.doi: 10. 1200/JCO.2004.12.188.

\section{How to cite this article?}

Dev R, Sharma P, Khan Y. Adenocarcinoma insitu presenting as extensive crazy paving pattern: HRCT findings and review of literature. Int J Med Res Rev 2018; 6(07):393-396. doi:10.17511/ijmrr.2018.i07.09. 